\title{
GERARD VIENNOT
}

\section{Quelques bases et familles basiques des algèbres de Lie libres commodes pour les calculs sur ordinateurs}

\author{
Mémoires de la S. M. F., tome 49-50 (1977), p. 201-209 \\ <http://www.numdam.org/item?id=MSMF_1977_49-50_201_0>
}

CC Mémoires de la S. M. F., 1977, tous droits réservés.

L'accès aux archives de la revue « Mémoires de la S. M. F. » (http://smf. emath.fr/Publications/Memoires/Presentation.html) implique l'accord avec les conditions générales d'utilisation (http://www.numdam.org/conditions). Toute utilisation commerciale ou impression systématique est constitutive d'une infraction pénale. Toute copie ou impression de ce fichier doit contenir la présente mention de copyright.

\section{Numdam}


Bull. Soc. math. France

Mémoire 49-50, 1977, p. 201-209

\section{QUELQUES BASES ET FAMILLES BASIQUES}

DES ALGEBRES DE LIE LIBRES

COMMODES POUR LES CALCULS SUR ORDINATEURS

- par Gérard VIENNOT

INTRODUCTION -

Le but de cet exposé est de définir une classe de bases et de familles basiques des algèbres de Lie libres e,t de montrer que leurs propriétés remarquables les rendent nettement plus pratiques pour les calculs sur ordinateurs que les bases classiques de Hall habituellement employées (voir par exemple (2)). L'une de ces bases est notamment utilisée par J. Michel pour le calcul des coefficients de $1 a$ série de Baker-Hausdorff́ et pour certains calculs relatifs au problème de Burnside et à la conjecture de Hughes (voir 1 'exposé à ces mêmes journées ( $\left.{ }^{8}\right)$ ). L'implémentation est faite en APL qui semble être un langage bien adapté à ces calculs et dont 1'intérêt a aussi été souligné au çurs de ces journées par J.F. Perrot (10). $\S 1$ - RAPPELS SUR LE CALCUL DES COMMUTATEURS ET LES ALGEBRES DE LIE LIBRES -

Ces quelques rappels élémentaires sont destinés au lecteur non spécialiste. Ils constituent aussi un préliminaire à 1'article de J. Michel à ces mêmes journées . Pour plus de détails, le lecteur se reportera à (l), (7) ou au chapitre 5 de $\left({ }^{9}\right)$.

Soit $X$ un ensemble non vide. Nous désignons par $M(X)$ (resp. $X^{+}$, resp. $X^{*}$ ) le magma libre (resp. demi-groupe libre, resp. monoïde libre) engendré par $x$, c'est-à-dire le système algébrique libre engendré par X et associé à une loi de composition (resp. loi associative, resp. loi associative avec élément neutre). Le groupe libre engendré par $X$ est noté $F(X)$.

Soit $\mathbb{K}$ un anneau commutatif unitaire. L'algèbre associative libre engendrée par $X$ à coefficients dans $\mathbb{K}$ (ou algèbre des polynômes en variables non commutatives $X)$ sera notée $\mathbb{K}<X>$. C'est un module libre de base $X^{*}$. L'algèbre de Lie libre engendrée par $X$ (à coefficients dans $\mathbb{K}$ ) sera notée $L(X)$ (ou $I_{\mathbb{K}}(X)$ s'il y a confusion).

Soit $\psi$ 1'application canonique consistant à " remplacer " les parenthèses d'un mot de $M(X)$ par le crochet de Lie $[\mathrm{u}, \mathrm{v}]=\mathrm{u} v-\mathrm{v}$. Par exemple :

$$
\psi(\mathrm{x},(\mathrm{x}, \mathrm{y}))=[\mathrm{x},[\mathrm{x}, \mathrm{y}]]=\mathrm{x}^{2} \mathrm{y}-2 \mathrm{x} \mathrm{y} \mathrm{x}+\mathrm{y} \mathrm{x}^{2} \in \mathbb{K}<\mathrm{x}>.
$$

Le module $\mathrm{L}(\mathrm{X})$ est un module libre et peut être identifié au sous-module formé des polynômes de Lie, c'est-à-dire les combinaisons linéaires d'éléments de $M(X)$ ) (ou alternants). La multiplication de $\mathrm{L}(\mathrm{X})$ est alors le crochet de Lie $[\mathrm{u}, \mathrm{v}]=\mathrm{u} \mathrm{v}-\mathrm{v} \mathrm{u}$ (qui est bien un polynôme de Lie lorsque $\mathrm{u}$ et $\mathrm{v}$ le sont). $\mathrm{L}^{\prime}$ algèbre enveloppante de $\mathbb{K}<\mathrm{X}>$ est $\mathrm{L}(\mathrm{X})$. 
Si $\mathcal{L}$ est une sous-algèbre de Lie libre de $\mathrm{L}(\mathrm{X})$, une famille basique de $\mathscr{L}$ est une famille d'éléments de $\mathrm{L}(\mathrm{X})$ engendrant librement $\mathscr{L}$ (en tant qu'algèbre de Lie).

Nous notons $X^{n}$ (resp. $M_{n}(X)$, resp. $L_{n}(X)$ ) $I^{\prime}$ ensemble des éléments de $X^{*}$ (resp. de $M(X)$, resp. éléments homogènes de $L(X)$ ) de longueur ou degré $n$.

La dimension du module libre $\mathrm{L}_{\mathrm{n}}(\mathrm{X})$ est donnée par les formules de Witt (avec $x$ fini de caldinal q):

$$
\begin{aligned}
& 1_{q}(n)=\sum_{d \prod_{n} \mu(d) q^{n / d}} \\
& \text { où } d \text { parcourt les diviseurs de } n \text { et } \mu \text { elln } 1 \text { a fonction de Moëbius habitue11e). }
\end{aligned}
$$

Calcul des commutateurs -

Nous donnons maintenant le lien entre les algèbres de Lie et le calcul dans les groupes.

Pour $\mathrm{u}$ et $\mathrm{v}$ éléments de $\mathrm{F}(\mathrm{X})$ notons $\{\mathrm{u}, \mathrm{v}\}=\mathrm{u} \mathrm{v} \mathrm{u}^{-1} \mathrm{v}^{-1}$ le commutateur de $u$ et $v$ (habitueliement noté aussi ( $u, v)$ ). Soit $\Phi$ l'application $M(X) \rightarrow F(X)$ consistant à remplacer les parenthèses d'un élément de $M(X)$ par des parenthèses de commutateurs et soit $F_{n}(X)$ le sous-groupe de $F(X)$ engendré par les commutateurs de degré $n$, c'est-à-dire $\Phi\left(M_{n}(X)\right)$. La suite $F_{n}(X)$ est appelée la suite centrale descendante du groupe $G=F(X)$. C'est un exemple de $\mathrm{N}$-suite, c'est-àdire une suite $\left(F_{n}\right)$ de sous-groupes distingués du groupe $G$ vérifiant les deux conditions :

$$
\begin{aligned}
& F_{1}=G \supseteq \ldots \supseteq F_{n} \supseteq F_{n+1} \supseteq \cdots \\
& \left\{F_{i}, F_{j}\right\} \subseteq F_{i+j}, \quad \forall i, j \geqslant 1
\end{aligned}
$$

(en désignant par $\left\{F_{i}, F_{j}\right\}$ le sous-groupe de $G$ engendré par les commutateurs $\{u, v\}, u \in F_{i}, v \in F_{j}$ ).

A toute N-suite $\left(F_{n}\right)$ d'un groupe $G$, on peut associer une $\mathbb{Z}$-algèbre de Lie $\mathscr{L}\left(F_{n}\right)$ de la façon suivante (voir $\left({ }^{7}\right)$ ): Le module sous-jacent est la somme directe des groupes abéliens libres $\mathrm{F}_{\mathrm{n}} / \mathrm{F}_{\mathrm{n}+1}$. En désignant par $\tilde{x}_{i}$ la classe de $x_{i} \epsilon F_{i}$ module $F_{i+1}$, on démontre que $\left\{x_{i}, x_{j}\right\}$ est un élément de $F_{i+j}$. dont la classe modulo $F_{i+j+1}$ ne dépend que de $\tilde{x}_{i}$ et $\tilde{x}_{j}$. On peut ainsi définir une applicatiọn bilinéaire

$$
F_{i} / F_{i+1} \times F_{j}+F_{j+1} \rightarrow F_{i+j} / F_{i+j+1}
$$

qui s'étend par linéarité au module $\mathcal{L}\left(F_{n}\right)$. Les identités remarquables existant entre commutateurs prouvent alors que $\mathscr{L}\left(F_{n}\right)$ est une algèbre de Lie.

En particulier lorsque 1 'on prend comme $\mathrm{N}$-suite la suite centrale descendante $\left(F_{n}(X)\right)$ du groupe libre $F(X)$, l'algèbre de Lie associée $\mathscr{L}\left(F_{n}\right)$ est alors 1'algèbre de Lie libre $\mathrm{L}_{\boldsymbol{Z}}(\mathrm{X})$. Les dimensions des groupes abéliens libres $\mathrm{F}_{\mathrm{n}} / \mathrm{F}_{\mathrm{n}+1}$ sont donc $1_{q}(n)$ et à une base d'alternants de $L_{n}(X)$ correspond ce qu'on appelle des commutateurs basiques de $F_{n}(X)$. 
Plongement du groupe libre dans le groupe des séries inversibles -

Un autre moyen de retrouver cette correspondance entre commutateurs et crochets de Lie est de plonger le groupe libre $F(X)$ dans le groupe $G(X)$ des séries inversibles de $\mathbb{K}<\langle X>>$, c'est-à-dire l'algèbre des séries à variables non commutatives $X$.et à coefficients dans $\mathbb{K}$. Soit $\rho 1^{\prime}$ application $F(X) \rightarrow G(X)$ définie par: $\forall x \in X, \rho(x)=\cdot 1+x$.

Alors $F(X)$ est isomorphe à $\rho(F(X))$. Notons $d(s)$ l'élément homogène de degré minimum $\geqslant 1$ de la série $s \in \rho(F(X))$. Alors la suite de sous-groupe de $F(X)$ définie par $F_{n}(X, \mathbb{K})=\{u \in F(X), d(\rho(u)) \geqslant n\}$ est une $N$-suite. En particulier si $\mathbb{K}=\mathbb{Z}$ on retrouve 1 a suite centrale descendante $F_{n}(X)$ et les modules $d \bullet \rho\left(F_{n}(X)\right)$ sont isomorphes aux modules $L_{n}(X)$. Lorsque $\mathbb{K}=\mathbf{z}_{n}$ on retrouve 1a $\mathrm{N}$-suite des groupes de dimension modulo $\mathrm{n}$.

Bases de Ha11 -

Nous rappelons enfin les classiques bases de Hall habituellement employées dans la pratique (5) et introduites par P. Hall en termes de commutateurs basiques dans le " collecting process " en (6.). Soit $H$ une partie totalement ordonnée de $M(X)$ vérifiant les trois conditions suivantes :

$-\left(\mathrm{Ha}_{1}\right) \quad \mathrm{X} \subseteq \mathrm{H}$

- $\left(\mathrm{Ha}_{2}\right) \forall \mathrm{h}=(\mathrm{u}, \mathrm{v}) \in \mathrm{M}(\mathrm{X}) \backslash \mathrm{X}, \mathrm{h} \in \mathrm{H} \Leftrightarrow\left\{\begin{array}{l}\mathrm{u} \in \mathrm{H}, \mathrm{v} \in \mathrm{H} \\ \mathrm{u}<\mathrm{v} \\ \mathrm{v} \in \mathrm{X} \text { ou } \mathrm{v}=\left(\mathrm{v}^{\prime}, \mathrm{v}^{\prime \prime}\right) \text { avec } \mathrm{v}^{\prime} \leqslant \mathrm{u}\end{array}\right.$

- $\left(\mathrm{Ha}_{3}^{\prime}\right) \forall \mathrm{u} \in \mathrm{H}, \forall \mathrm{v} \in \mathrm{H},|\mathrm{u}|<|\mathrm{v}| \Rightarrow \mathrm{u}<\mathrm{v}(|\mathrm{u}|$ est la longueur de $\mathrm{u})$

Alors la famille $\{\psi(h), h \in H\}$ est une base de $L(X)$ appelée base de Hall de $L(X)$.

Les bases des algèbres de Lie libres sont non seulement utiles dans les calculs de théorie combinatoire des groupes, mais aussi dans d'autres domaines comme par exemple la rēsolution d'équations différentielles linéaires ou en mécanique quantique (théorie des perturbations). Signalons enfin le calcul des coefficients de la célèbre série (en variables non commutatives) $z=\log \left(e^{x} e^{y}\right)=x+y+\frac{1}{2}(x, y)$ $+\ldots$ appelée série de Baker-Hausdorff et qui est en fait une somme d'alternants de $L_{\mathbb{Q}}(\mathrm{x}, \mathrm{y})$.

$\S 2$ - FACTORISATIONS REGULIERES DES MONOIDES LIBRES ET BASES DE CHEN-FOX-LYNDONSIRSOV -

Les bases que nous décrivons sont définies comme cas particuliers de la construction générale suivante :

Définition 2-1 -

Une partie $F$ totalement ordonnée de mots de $\mathrm{x}^{+}$est appelée factorisation régulière complète ssion a les trois conditions : 
(i) $\quad \mathrm{X} \subseteq \mathrm{F}$

(ii) $\forall u, v \in F, u<v \Rightarrow u v \in F$ et $u<u v<v$

(iii) $\forall f \in F \backslash x, \exists u, v \in F$ avec $u<v$ tel que $f=u v$

Exemple 2.1 -

Soit $\mathrm{X}=\{\mathrm{x}, \mathrm{y}\}$. Nous donnons une construction possible des mots de longueur $\leqslant 5$ de $F$. Les éléments de $F \cap X^{n}(n \leqslant 5)$ sont écrits en ligne et ordonnés de gauche à droite.

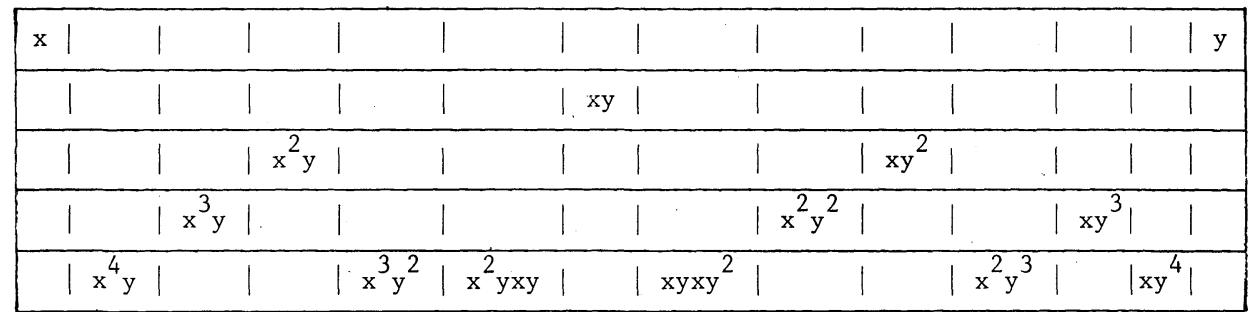

On trouvera une construction générale des factorisations régulières complètes dans $(14)$.

Pour une factorisation régulière complète $F$ de $X$, soit $\lambda 1$ 'application $F \rightarrow L(X)$ définie de la façon suivante :

$-\forall \mathrm{x} \in \mathrm{X} \quad \lambda \mathrm{x}=\mathrm{x}$

- pour $f \in F \backslash X$, on peut toujours écrire $f=u v$ avec $u \in F$ et $v \in X^{+}$. Soit $f^{\prime}$ le plus long des facteurs gauches de $f$ tel que :

$$
f=f^{\prime} f^{\prime \prime} \text { avec } f^{\prime} \in F \text { et } f^{\prime \prime} \in X^{+} \text {. }
$$

Alors les conditions (i), (ii), (iii) de la définition 2.1 impliquent que $f^{\prime \prime} \in$ F. On peut donc définir par récurrence sur les longueurs des mots de $\mathrm{F}$ :

$$
\lambda f=\left[\lambda f^{\prime}, \lambda f^{\prime \prime}\right]
$$

PROPOSITION $2.1-$

Soit $F$ une factorisaiton régulière complète du monoïde libre $X$. Alors 1a famille $\{\lambda(f), f \in F\}$ est une base de $L(X)$.

Base de Chen-Fox-Lyndon-Šrirsov -

Soit $\leqslant$ un ordre lexicographique sur $X$ et soit $F$ l'ensemble des mots de $x^{+}$ qui sont minimum (pour 1'ordre $\leqslant$ ) dans leur classe de conjugaison, c'est-à-dire : $F=\left\{f \in X, f=u v, u \in X^{+}, v \in X^{+} \Rightarrow f<v u\right\}$

Alors les propriétés de l'ordre lexicographique impliquent que $F$ est une factorisation régulière complète (en l'ordonnant par 1 'ordre lexicographique). La proposition 2.1 permet de retrouver la base $\{\lambda(f), f \in F\}$ introduite en $(3)$, $(12)$ et $\left({ }^{13}\right)$ et que nous appelons base de Chen-Fox-Lyndon-Širšov. Les mots de $\mathrm{F}$ sont appelés lexicographiques standards. 
Exemple 2.2 -

Soit $\mathrm{X}=\{\mathrm{x}, \mathrm{y}\}$ avec ' $\mathrm{x}<\mathrm{y}$. Les mots de longueur 5 de $\mathrm{F}$ sont :

$$
\left\{x^{4} y, x^{3} y^{2}, x^{2} y x y, x^{2} y^{3}, x y x y^{2}, x y^{4}\right\}
$$

Les éléments de degré 5 de la base de Chen-Fox-Lyndon-Sirsov sonț donc :

$$
\begin{aligned}
& \lambda\left(x^{4} y\right)=[x,[x,[x,[x, y]]]] \\
& \lambda\left(x^{3} y^{2}\right)=[[x,[x,[x, y]]], y] \\
& \lambda\left(x^{2} y x y\right)=[[x,[x, y]],[x, y]] \\
& \lambda\left(x^{2} y^{3}\right)=[[[x,[x, y]], y], y] \\
& \lambda\left(x^{4}\right)^{\prime}=[[[[x, y], y], y], y] \\
& \lambda\left(x y x y^{2}\right)=[[x, y],[[x, y], y]]
\end{aligned}
$$

La propriété fondamentale rendant cette base très commode pour les calculs est 1a suivante :

PROPOSITION $2.2-$

Soit F l'ensemble des mots lexicographiques standards de $X^{*}$ et soit $\omega \underline{1 a}$ projection de $L(X)$ sur le sous-module libre de $Z<X>$ de base $F$. Alors $\omega$ est un isomorphisme de module. Les matrices associées à 1 a restriction de $\omega$ à $L_{n}(X)$ exprimant les images par $\omega$ de $\lambda\left(F \cap X^{n}\right)$ relativement à la base $F \cap X^{n}$ sont triangulaires, à coefficients entiers avec des 1 sur 1a diagonale.

Cette propriété se montre facilement en remarquant que pour tout $f \in \mathrm{F}$, le polynôme de Lie $\lambda(f)$, s'écrit $\lambda(f)=f+\lambda^{\prime}(f)$ avec $\lambda^{\prime}(f)$ somme de monômes de $\boldsymbol{Z}<\mathrm{X}>$ tous strictement supérieurs pour l'ordre lexicographique à f.

En fait la proposition 2.2 est vraie en général pour les bases associées aux factorisations régulières complètes des monoïdes libres. Nous verrons un autre exemple au paragraphe suivant avec la base que nous appelons base de Spitzer-Foata, nouvelle base introduite par 1'auteur en (14). Remarquons que le projecteur analogue associé à la base de Hall n'est même pas un isomorphisme.

Application aux calculs sur ordinateurs -

Un programme APL permet de calculer les mots lexicographiques standards de degré donné. On peut alors calculer l'application $\lambda$ associée, puis les matrices de la proposition 2.2 avec des fonctions manipulant en APL les polynômes non commutatifs. Une application est le calcul des coefficients de la série de Baker-Hausdorff relativement à la base de Chen-Fox-Lyndon-Sirsov. Il suffit de connaître les coefficients de cette série de $\Phi<<X>>$, considérée comme somme de mots de $X^{*}$ et qui sont donnés par des formules dues à Goldberg. On ne retient dans ces coefficients uniquement ceux relatifs aux mots lexicographiques standards. Pour en déduire ceux relativement à $1 \mathrm{a}$ base de Chen-Fox̣-Lyndon-Širšov il suffit d'inverser les matrices triangulaires de 1 a proposition 2.2, qui sont encore des matrices à coefficients entiers. On peut calculer indépendemment chaque composante bihomogène de degré donné en $x$ et en $y$. 
Par exemple la matrice associée à la restriction de $\omega$ à $F \cap \mathrm{X}^{5}$ se décompose en les quatre sous-matrices triangulaires suivantes :

\begin{tabular}{|c|c|c|c|c|c|c|}
\hline & $\lambda\left(x^{4} y\right)$ & $\lambda\left(x^{3} y^{2}\right)$ & $\lambda\left(x^{2} y x y\right)$ & $\lambda\left(x^{2} y^{3}\right)$ & $\lambda\left(\mathrm{xyxy}^{2}\right)$ & $\lambda\left(x y^{4}\right)$ \\
\hline$x^{4} y$ & 1 & 0 & 0 & 0 & 0 & 0 \\
\hline$x^{3} y^{2}$ & $--\overline{0}^{-}$ & 1 & $0^{-\cdots}$ & 0 & 0 & 0 \\
\hline$x^{2} \cdot y x y$ & 0 & -3 & 1 & 0 & 0 & 0 \\
\hline$x^{2} y^{3}$ & 0 & $-\cdots$ & 1 & $-\overline{1}$ & ----- & 0 \\
\hline$x y x y^{2}$ & 0 & 0 & 0 & -2 & 1 & $\underline{0}$ \\
\hline$x y^{4}$ & 0 & 0 & 0 & 0 & 0 & 1 \\
\hline
\end{tabular}

En fait la proposition 2.1 n'est qu'un corollaire d'une théorie générale des factorisation's des monö̈des libres, notions introduites par M.P. Shützenberger (11) et développées dans la thèse de 1 'auteur $(14)$. Une factorisation complète du monoïde libre $X^{*}$ est un ensemble $F$ de mots totalement ordonnés tels que tout mot $f \in x^{+}$se factorise de manière unique $f=f_{1} \ldots f_{p}$ avec $p \geqslant 1, f_{i} \epsilon F$, $\mathrm{f}_{1} \geqslant \ldots \geqslant \mathrm{f}_{\mathrm{p}}$. On a alors la généralisation maximum possible des bases de Hall: (en notant $\delta: M(X) \rightarrow X^{*} I^{\prime}$ application canonique de déparenthésage).

PROPOSITION $2.3-$

Soit $H$ une partie totalement ordonnée de $M(X)$ vérifiant $\left(\mathrm{Ha}_{1}\right)$ et $\left(\mathrm{Ha}_{2}\right)$ Alors la famille $\{\psi(h), h \in H\}$ est une base de $L(X)$ sśi $\delta H$, ordonné par l'ordre correspondant de $\mathrm{H}$, est une factorisation complète de $\mathrm{X}^{*}$, ce qui équivaut à $1 \mathrm{a}$ condition :

$$
\left(\mathrm{Ha}_{3}\right) \quad \forall \mathrm{u}, \mathrm{v} \in \mathrm{H} \quad(\mathrm{u}, \mathrm{v}) \in \mathrm{H} \Rightarrow \mathrm{u}<(\mathrm{u}, \mathrm{v})
$$

Les factorisations complètes régulières introduites dans cet exposé sont alors exactement les factorisations telles qu'il existe un ensemble $\mathrm{H}$ vérifiant les conditions. $\left(\mathrm{Ha}_{1}\right),\left(\mathrm{Ha}_{2}\right)$ et $\left(\mathrm{Ha}_{3}\right)$, ainsi qu'un ensemble $\mathrm{H}^{\prime}$ vérifiant les conditions duales. La base associée par la proposition 2.1 est alors $\psi(\mathrm{H})$.

\section{$\S 3$ - FAMILLES BASIQUES ET BASE DE SPITZER-FOATA -}

On peut vérifięr sur ordinateur qu'il n'existe pas de base de $L(X)$ vérifiant les conditions de la proposition 2.2 et telles que les matrices associées soient les matrices unités. De fait, il n'existe pas de base " canonique " de L(X). Par contre il existe certaines décompositions de $\mathrm{L}(\mathrm{X})$ en somme directe (en tant que module) de sous-algèbres de Lie libres. Nous donnons ici des procédés de calcul rapide des familles basiques de ces sous-algèbres de Lie. Ceux-ci reposent sur la notion générale de factorisation de monoïde libre due à M.P. Schützenberger (11) et développée par 1 'auteur $(14)$. 
Définition 3.1 -

Une factorisation $F=\left(Y_{j}, j \in J\right)$ du monoide libre $X^{*}$ est une famille de parties de $X^{+}$indexée par un ensemble totalement ordonné $J$ et telle que tout mot $f$ de $X^{+}$se factorise de manière unique

Exemple 3.1 -

$$
\mathrm{f}=\mathrm{f}_{1} \ldots \mathrm{f}_{\mathrm{p}} \text { avec } \mathrm{p} \geqslant 1, \mathrm{f}_{\mathrm{i}} \in \mathrm{Y}_{\mathrm{j}_{\mathrm{i}}}, \mathrm{j}_{1} \geqslant \ldots \geqslant \mathrm{j}_{\mathrm{p}} \text {. }
$$

Exemple $3.2-$

$$
\mathrm{X}=\mathrm{b}+\mathrm{Y}, \mathrm{J}=\{1,2\}, \mathrm{Y}_{1}=\underset{\mathrm{i}}{\mathrm{U}} \mathrm{b}^{i} \mathrm{Y}, \mathrm{Y}_{2}=\{\mathrm{b}\}
$$

Lẹs factorisations complètes régulières de la définition 2.1 sont des factorisations avec $F=J$ et $Y_{j}=\{j\}$. Plus généralement une factorisation complète est une factorisation telle que chaque $Y_{j} n^{r}$ ait qu'un seul élément.

On peut généraliser les propriétés de paragraphe 2 en définissant la classe des factorisations régulières de $X^{*}$ comme les factorisations vérifiant :

Définition 3.2 -

Une factorisation $\mathscr{T}^{\prime}=\left(Y_{j}, j \in J\right)$ de $X$ est dire régulière ssielle vérifie 1 'une des deux conditions équivalentes suivantes :

(i) $\forall u \in Y_{j_{1}}, \forall v \in Y_{j_{2}}, j_{1}<j_{2} \Rightarrow u v \in \underset{j}{u} Y_{j}$.

(ii) $\forall u \in Y_{j_{1}}, \forall v \in Y_{j_{2}}, u v \in \bigcup_{j}^{U} Y_{j} \Rightarrow u v \in Y_{k}$ avec $. j_{1}<k<j_{2}$

Par le même procédé de " crochetage " des mots du paragraphe 2 (en remplaçant $F$ par $\left.\operatorname{Cont}(\mathscr{G})=\mathscr{J}_{J} \mathrm{Y}_{\mathrm{j}}\right)$, on définit une application $\lambda: \operatorname{Cont}(\mathscr{T}) \rightarrow \mathrm{L}(\mathrm{X})$ telle que chaque $\lambda\left(\mathrm{Y}_{\mathrm{j}}\right)$ soit une famille d'alternants engendrant librement 1a sous-a1algèbre de Lie $\mathcal{Z}\left(\lambda\left(\mathrm{Y}_{\mathrm{j}}\right)\right)$ de $\mathrm{L}(\mathrm{X})$, qu'elle engendre. De plus $\mathrm{L}(\mathrm{X})$ peut être identifié en tant que module à la somme directe :

$$
L(X) \simeq \underset{j}{\oplus} L\left(\lambda\left(Y_{j}\right)\right.
$$

L'exemple fondamental de ce paragraphe est la factorisation dite de Spitzer sur deux lettres $\mathrm{X}=\{\mathrm{x}, \mathrm{y}\}$, qui factorise " canoniquement " $\mathrm{L}(\mathrm{X})$ et d'où est issue la nouvelle base de Spitzer-Foata.

Pour $x \in X$ nous notons $|\dot{u}|_{x}$ le degré en $x$ du mot $u$ de $X^{*}$ ou de 1'a1ternant, $u$ de $L(X)$. Soit $J$ l'ensemble des couples $(p, q)$ de nombres premiers entre eux, augmenté du 0 et ordonné par 1 'ordre induit des rationnels $q / p$.

Notons $Y_{0}=\{x\}$ et pour $(p, q) \in J \backslash\{0\}$

$\mathrm{Y}_{(\mathrm{p} ; \mathrm{q})}=\left\{\mathrm{f} \in \mathrm{X}^{+},|\mathrm{f}|_{\mathrm{y}} /|\mathrm{f}|_{\mathrm{x}}=\mathrm{q} / \mathrm{p}\right.$ et $\left.\forall \mathrm{u}, \mathrm{v} \in \mathrm{X}^{+}, \mathrm{f}=\mathrm{u} \mathrm{v},|\mathrm{u}|_{\mathrm{y}} /|\mathrm{u}|_{\mathrm{x}}<\mathrm{q} / \mathrm{p}\right\}$

On peut exprimer géométriquement cette définition en associant à tout mot de $\mathrm{X}^{+}$un " chemin minimal " de $\mathbb{N} \times \mathbb{N}$ : chaque occurence de $\mathrm{x}$ (resp. y) correspond à un "pas ". horizontal (resp. vertical).

Exemple 3.3 -

Au mot $f=$ xyxxyxyyy correspond le chemin minimal suivant : 


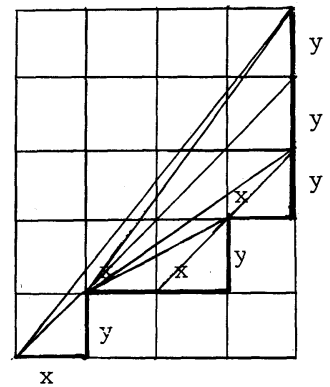

Les mots de $Y\left({ }_{p, q}\right)$ correspondent aux chemins minimaux situés strictement (sauf aux extrémités) sous $1 \mathrm{a}$ droite de pente $\mathrm{q} / \mathrm{p}$ passant par le point $(0,0)$. On vérifie que $\mathscr{F}=\left(\mathrm{Y}_{\mathrm{j}}, \mathrm{j} \in \mathrm{J}\right)$ est une factorisation régulière.

Sur la figure on peut " lire " le parenthésage associé au mot $f \in \operatorname{Cont}(\mathscr{G})=\bigcup_{j} Y_{j}$. Ainsi celui de $f=$ xyxxyxyyy $\in Y_{5 / 4}$ est : $\lambda(f)=[[x, y],[[[x,[x, y]],[x, y]], y], y]]$

Soit d'autre part $L_{(p, q)}$ la sous-algèbre de Lie libre de $L(X)$ constituée des combinaisons linéaires d'alternants u tels que $|u|_{y} /|u|_{x}=q / p$, et soit $L_{0}=\{x\}$. Il est clair que $L(X)$ est somme directe des $L_{j}, j \in J$. Il est nettement moins évident que :

$\underline{\text { PROPOSITION } 3.1-}$

$\lambda\left(Y_{j}\right)$ est une famille basique de l'algèbre de Lie $L_{j}$ et donc le module $L(X)$ est isomorphe à la somme directe $\oplus \mathrm{H}\left(\lambda\left(\mathrm{Y}_{\mathrm{j}}\right)\right)$.

Dans un certain sens, ces familles basiques possèdent encore la propriété de la proposition 2.2. Elles sont donc particulièrement commodes pour les calculs sur ordinateurs. Le calcul des matrices triangulaires s'obtient par des calculs de pentes de chemins minimaux comme indiqués sur la figure. Pour avoir une base de $\mathrm{L}(\mathrm{X})$ il suffit de prendre une base dans chaque algèbre de Lie $L\left(\lambda\left(\mathrm{Y}_{j}\right)\right)$. L'avantage est que quelque soit le choix de ces bases, les coefficients d'un polynôme de Lie $\mathrm{L}(\mathrm{X})$ relativement aux alternants de $\bigcup_{j} \lambda\left(Y_{j}\right)$ ne changent pas et sont en un certain sens " canoniques ". En particulier ${ }^{j} i 1^{j}$ 'on prend dans chaque $Y_{j}^{*}$ sousmonoïde libre de $X^{*}$, l'ordre lexicographique défini en ordonnant $Y_{j}$ lexicographiquement par $\mathrm{x}<\mathrm{y}$, puis en définissant la factorisation $\mathscr{F}_{\mathrm{j}} \mathrm{de} \mathrm{Y}_{\mathrm{j}}^{*} \operatorname{des} \operatorname{mots}$ lexicographiques standards, on obtient par " composition " de la factorisation $\mathscr{F}=\left(\mathrm{Y}_{\mathrm{j}}, \mathrm{j} \in \mathrm{J}\right)$ avec chacune des factorisations $\mathscr{F}_{\mathrm{j}}$ une factorisation complète régulière $F$ de $X$. La base associée de $L(X)$ est la base que nous appelons base de Spitzer-Foata (le fait d'être une base avait été conjecturé par D. Foata). Cette base est obtenue aussi comme la réunion des différentes bases de 
Chen-Fox-Lyndon-Sirsov relativement à chaque sous-algèbre de Lie libre $L\left(\lambda\left(Y_{j}\right)\right)$. PROPOSITION $3.2^{\circ}-$

La base de Spitzer-Foata vérifie 1a propriété de 1a proposition 2.2.

Cette base n'a pas encore été utilisée en APL.

Il serait peut être intéressant de calculer les coefficients canoniques de la série de Baker-Hausdorff relativement aux alternants de $\bigcup_{j}\left(Y_{j}\right)$. On sait déjà que ceux des alternants associés aux mots ${ }_{x} \mathrm{p}^{\mathrm{q}}$ sont des combinaisons de nombres de Bernoul1i.

\section{BILBIOGRAPHIE}

(1) BOURBAKI N.- Groupes et algèbres de Lie.- Ch. 2, Algèbre de Lie 1ibres.Paris, Hermann, (1972) (Act. scient. et ind., 1349; Bour. 37)

(2) CANNON J-J.- Some combinatorial and symbol manipulation programs in group theory.- Proc. Int. Conf. Theory of groups.- p. 199-203.

(3) CHEN K-T., FOX R-H. and LYNDON F-C.- Free differential calculus IV, The quotient groups of the lower central series.- Ann. of Math.- t.68 (1958) p. $81-95$.

$\left({ }^{4}\right)$ FOATA D. - Etude algébrique de certains problèmes d'analyse combinatoire et du calcul des probabilités.- Pub. Inst. Statist. Univ. Paris.) t. 14; (1965) p. 81-241 (Thèse Sc. Math., Paris, 1965).

(5) HALL M-J.- A basis for free Lie rings and higher commtators in free groups.Proc. Amer. Math. Soc.- t. 1 (1950) p. 575-581.

(6) HALL P.- A contribution to the theory of groups of prime-power order.- Proc. London Math. Soc.- t. 36 (1933) p. 29-95.

(7) LAZARD M. - Sur les groupes nilpotents et les anneaux de Lie.- Ann. Sci. ENS.3, 71, (1954) p. 101-190 (Thèse Sc. Math. Paris).

(8) MICHEL J.- Journées algorithmiques.- Astérisque n 38-39, (1976) p. 139-148

(9) MAGNUS W., KARASS A., SOLITAR D.- Combinatorial group theory.- New-York.Interscience Publishers.- (1966).

(10) PERROT J-F.- Exposé à ces mêmes journées.

(11) SCHUTZENBERGER M-P.- On a factorisation of free monoids.- Proc. Amer. Math. Soc.- t. 16, (1965) p. 21-24.

(12) ŠIRŠOV A-I.- On free Lie rings.- Math. Sbornik, N. S.- 87, 45, (1958) p. $113-122$

(13) ŠIRŠOV A-I.- On bases for free Lie algebra.- Algebra i Logika Sém., t. 1; (1962) p. 14-19.

(14) VIENNOT G.- Factorisations des monö̈des libres et algèbres de Lie 1ibres.(Thèse Sc. Math.- Paris, (1974) à paraître).

Gérard VIENNOT

E.N.S. Centre de Mathématiques

45, rue d'Ulm

PARIS 5ème 